\title{
La construcción de coaliciones para combatir la corrupción
}

\section{M aría González de Asís*}

\section{Introducción}

La construcción de coaliciones entre la sociedad civil y el gobierno es un paso importante en la lucha contra la corrupción. Abriendo canales a través de los cuales la sociedad civil y los stakeholders (accionistas) del gobierno pueden exigir mayor fiabilidad el uno del otro, este acercamiento puede generar y mantener una dinámica ciudadano-gobierno que refuerce sustancialmente las reformas. Además, incorporando los instrumentos para el diagnóstico y los talleres con una amplia base en el proceso de construcción de la coalición, ayuda a identificar las oportunidades y las prioridades para los esfuerzos de la reforma.

La corrupción no es un asunto nuevo. Sin embargo, durante losúltimosaños, los estudiosos, así como los gobiernos y los miembros de la sociedad civil, han empezado a darse cuenta de que esta relación distorsiona la distribución de los recursos. Este conocimiento ha impulsado a las sociedades civiles de muchos países a presionar a sus gobiernos para que haya un cambio, y ha llevado a muchos gobiernos a asumir reformas a largo plazo de las instituciones del sector público. M uchos gobiernos han pedido apoyo (donor support) para diseñar una estrategia anti-corrupción efectiva, expresado su disposición para implicar a diferentes stakeholders en el proceso ${ }^{1}$.

El Instituto del Banco Mundial (WBI) está respondiendo a un cada vez mayor número de peticiones para proporcionar ayuda para diseñar una estrategia participativa y comprensible para luchar contra la corrupción. En esta estrategia, el Instituto del Banco Munidal se esfuerza para asegurar el compromiso activo de la sociedad civil. Esto conlleva el trabajar para mejorar la comprensión y el apoyo para la agenda de reforma. La estrategia necesita de rigurosos instrumentos para el diagnóstico y llamadas a la formación de una comisión de iniciativas compuesta por la sociedad civil y por los representantes del gobierno. Además, la estrategia promueve las fuerzas de las tareas, de los grupos de trabajo, y de los talleres nacionales para discutir las reformas actuales. Estas actividades están enfocadas en áreas tales como la judicial, la de gestión, las aduanas o los medios de comunicación, cada uno de los cuales arrastra a diferentes stakeholders al proceso.

Las estrategias anti-corrupción tienen distintas vertientes y se integran bien en la Estrategia de Asistencia de París. Se llevan a cabo en estrecha cooperación con la sociedad civil, el gobierno, y otros donors. Un requisito previo para iniciar un programa es la existencia de una voluntad política real que permita a la sociedad civil y al gobierno llevar a cabo las reformas juntos. Aunque el Instituto del Banco M undial trabaja en muchos países, este trabajo se basa principalmente de los programas en Paraguay y Bolivia para ilustrar el trabajo del Instituo del Banco M undial en la lucha contra la corrupción.

\section{Expresando voluntad política}

Cuando un gobierno pide apoyo para diseñar un programa para luchar contra la corrupción, el WBI envía una misión para que se reúna con el alcalde y con los miembros de la sociedad civil, para asesorar acerca de la situación política en el país. Dichas peticiones suelen ocurrir cuando existe una oportunidad para la reforma, debido a elecciones o a la caída de un gobierno. 
La presencia de un reformador de alto nivel en el gobierno es una precondición para la ayuda del WBI. Una vez situado éste al frente de la reforma, sin embargo, no se podrá contar, durante el proceso, con un líder tan comprometido. Por lo tanto se necesitan otros stakeholders para apoyar el programa, y para presionar en ciertas ocasiones para mantener la voluntad política. Para que el proceso resulte créble y sostenible, los stake holders de la sociedad civil tienen que estar implicados desde el principio. Los stakeholders también necesitan incluir un rango suficientemente amplio de la población, como para soportar los cambios en la voluntad política, y para protegerse de la politización del proceso.

En Paraguay, por ejemplo, el mandato del Presidente González M acchi contribuyó a dar importancia a las reformas anticorrupción y a fomentar un proceso participativo. Después de jurar el cargo en marzo de 1999², pidió la asistencia del Banco para conducir estudios sobre la corrupción para diagnosticar y para diseñar un plan de acción anti-corrupción efectivo para la reforma del sector público. En mayo de 1999, el Banco Mundial se reunió con el gobierno paraguayo y con miembros de la sociedad civil y, consecuentemente, pusieron en marcha un programa comprensible dirigido por una coalición de gobierno y por los stakeholders de la sociedad civil. Su nuevo gobierno abrió una ventana para la oportunidad de diseñar un programa participativo para luchar contra la corrupción, aun cuando se ocupaba de un creciente déficit fiscal, de una economía enlodazada en una profunda recesión y de un colapso en las exportaciones.

En contraste, en Bolivia el proceso de reforma se empezó desde arriba, con poca implicación de la sociedad civil. El Presidente Banzer también pidió ayuda al Banco Mundial para desarrollar una estrategia de integridad nacional, pero se mostraba reticente a abrir completamente el proceso a los stakeholders de fuera del gobierno. Aunque al gunos miembros de la sociedad civil revisaron el plan de acción preparado por el gobierno, éste carecía de credibilidad debido a quela sociedad civil no estaba implicada en su desarrollo.

\section{Creando la Comisión de Iniciativas}

Para desarrollar estos programas, la Comisión de Iniciativas se establece generalmente para coordinar todo el trabajo anticorrupción en el país, incluyendo el trabajo de distintos donors. Los equipos del W BI trabajan con representantes de la sociedad civil y del gobierno para recibir consejo acerca de cómo debería estar compuesta la Comisión de Iniciativas. Durante las reuniones, el equipo presenta ejemplos de diferentes países, incluyendo el criterio que se utiliza para elegir a los miembros. Entonces, se debaten los miembros potenciales, se prueban y se decide sobre ellos, hasta que la reunión genera una lista de unas diez o quince personas.

La Comisión de Iniciativas está encabezada por un oficial de alto nivel del gobierno, e incluye a todos los miembros relevantes del gobierno y de la sociedad civil. En Bolivia, por ejemplo, la Comisión de Iniciativas está encabezada por el Vicepresidente, y compuesta por el M inistro de Aduanas, el M inistro de Justicia, el M inistro de la Presidencia, el Contralor, el General Auditor, la Corte Suprema, el Congreso, el sector privado, la iglesia y los medios de comunicación. En Paraguay, la Comisión de Iniciativas está encabezada por el M inistro de la Presidencia, y compuesto por el M inistro de Interior, el M inistro de Aduanas, el M inistro de Economía, el Asesor Político del Presidente, el Contralor de la República, la Corte Suprema, los partidos políticos, los medios de comunicación, las O N Gs, la juventud, la iglesia y el sector privado. Sin embargo, aś como en Bolivia la Comisión de Iniciativas está ampliamente dominada por miembros del gobierno, en Paraguay está más equilibrada entre los miembros del gobierno y los de la sociedad civil y tiene una gran credibilidad en todo el país.

La Comisión de Iniciativas se reúne regularmente para proponer una estrategia anti-corrupción, que debería incluir tanto un acercamiento a largo plazo, como un plan de acción a corto plazo concreto. El plan de acción está enfocado hacia la reforma institucional, y detalla las responsabilidades y el momento para cada acción. Los resultados del estudio para el diagnóstico sirven como base para el trabajo de la Comisión de Iniciativas, ayudando a identificar los problemas y los puntos débiles del sector público, y las principales dificultades en el desarrollo empresarial. Los resultados del estudio también ayudan a priorizar las reformas propuestas.

\section{Gestionando los estudios}

La extensión y la naturaleza de la corrupción en un país se miden a través de estudios y otros instrumentos para el diagnóstico. M edir la corrupción ayuda a establecer prioridades para la reforma, identificando las actividades y agencias donde se concentra la corrupción. Se utiliza la evidencia concreta para despersonalizar el debate sobre la corrupción, para cambiar el enfoque hacia temas más sustanciales, y para educar al público acerca de los costes económicos y social es de la corrupción. También establece una línea de referencia contra la que luego medir los éxitos 
o fracasos de la reforma. Los estudios para el diagnóstico son, por lo tanto, una parte integral para construir el consenso y para diseñar programas anti-corrupción.

Para iniciar el diagnóstico, el equipo se reúne con compañías que son capaces de dirigir los estudios sobre los funcionarios públicos, los ciudadanos y las empresas. Durante esta reunión, se explican con detalle el rango, los objetivos y la naturaleza de los estudios, y se proporciona más información, para que las compañías puedan presentar propuestas técnicas y financieras. La identificación de una compañía independiente y respetable es crucial para mantener la credibilidad de los resultados.

Los resultados de los estudios identifican los principales problemas de gobierno en los marcos seleccionados de instituciones nacionales, regionales y municipales. Estas instituciones están implicadas en la producción de servicios públicos, tales como la ley, la sanidad, la educación, las aduanas y la recogida de impuestos. Los estudios para el diagnóstico son la fuente principal de información cualitativa y cuantitativa para hacer recomendacioners políticas sobre las reformas anti-corrupción.

Los estudios muestran normalmente la presencia de prácticas sistémicas corruptas en áreas muy específicas del sector público. Estos resultados se corroboran generalmente, por estudios previos de donor, que revisan la infraestructura institucional del pás. Tales estudios incluyen consideraciones de cómo el clientelismo político y del patronato impiden la efectividad de varias agencias.

Se dirigieron varios estudios de diagnóstico en Bolivia durante 1998 y 1999. El WBI, trabajando en colaboración con la oficina del Vicepresidente, organizó un estudio nacional sobre las unidades familiares (6.851), un estudio sobre el sector privado (de 1.700 compañías), y un estudio sobre los funcionarios públicos (de 1.250 oficiales en 110 instituciones públicas). El trabajo, que fue sufragado con fondos noruegos, lo realizó la CIET Internacional. Los dos primeros estudios recogieron información sobre las percepciones de las compañías y ciudadanos acerca de la corrupción donde existen puntos de contacto con el público. Los estudios identificaron áreas institucionales donde la corrupción se percibe más atrincherada, incluyendo la polícía nacional, las aduanas, la administración de justicia, las instituciones que emiten los certificados de nacimiento, los títulos de propiedad y los documentos nacionales de identidad, y los gobiernos municipales, especialmente dentro de la región del eje central del pais.

El estudio sobre los funcionarios públicos recogió información sobre el cumplimiento del servicio, sobre la práctica de la gestión interna y sobre el entorno institucional de las agencias públicas. Los resultados del estudio muestran que las superintendencias, el Ministerio de la Presidencia, el Banco Central, el M inisterio de Gobierno, y la Corte Electoral, sobrepasan a otras instituciones nacionales con su calidad superior de servicio y sus bajos niveles de corrupción, mientras que la administración de impuestos, los departamentos de sanidad, las aduanas y la policía nacional exhiben cumplimientos decepcionantes de sus servicios.

Un análisis profundo del entorno institucional en el sector público boliviano revela que ni el relativo éxito, ni el relativo fracaso de las instituciones públicas son coincidencia. D etrás de cada historia de éxito, existe un sólido estilo gubernamental, apoyado por un reclutamiento basado en el mérito y en sistemas de promoción; por el aislamiento del entrometimiento e interferencia política; por las reglas y procesos internos que son simples, claros, estables y están bien especificados; por los mecanismos de fiabilidad que están bien establecidos y estrictamente impuestos; por la abierta y transparente toma de decisiones que permite la delegación de autoridad y la participación del personal en el proceso de toma de decisión; por la gestión del servicio basada en el cumplimiento, que está abierta a la retroalimentación y a las críticas por parte de los usuarios; y por los recursos apropiados para proporcionar una cantidad suficiente de servicios de alta calidad.

En Paraguay, estos tres tipos de estudio se realizaron también durante 1999: un estudio nacional de unidades familiares (2.499), un estudio sobre el sector público (de 553 compañías), y un estudio sobre los funcionarios públicos (1.451). Los primeros dos estudios recogieron información de la percepción de las compañías y de los ciudadanos acerca de la corrupción en determinadas áreas del sector público, mientras que el estudio de los funcionarios públicos se enfocó en el ejercicio del trabajo y en la gestión interna de las instituciones públicas. Los resultados de los estudios muestran que el Ministerio de Educación, la Presidencia, el Registro Civil, el servicio postal, y la compañía de petróleo, son mucho más eficientes a la hora de erradicar la corrupción, mientras que las aduanas, las compañías detelecomunicaciones, la Administración de la Seguridad Social, el M inisterio de Economía y el Contralor están percibidos como las instituciones más corruptas.

Los resultados también muestran que la corrupción tiene un significativo impacto negativo en la calidad y accesibilidad del servicio, afectando desproporcionadamente a las pequeñas empresas y a los pobres. Al igual que los resultados de los estudios en Bolivia, las comparaciones entre las instituciones con un alto cumplimiento y aquellos con uno bajo, revelan que las instituciones con un alto cumplimiento tienden a estar menos politizadas, a ser más meritocráticas, más transparentes en la toma de decisiones, con una producción de servicios más orientada hacia el consumidor, y más rico en recursos físicos y financieros. También han conseguido domar la excesiva discrecionalidad imponiendo reglas simples, bien especificadas y estables, a la vez que potencian la participación del personal en el proceso de toma de decisiones y que permiten la difusión vertical y horizontal de la responsabilidad dentro de la jerarquía. 


\section{Creando subcomisiones, dirigiendo programas y proporcionando entrenamiento}

Los resultados de los estudios identifican áreas prioritarias para lareforma, como, por ejemplo, laadministración dejusticia, lagestión y las aduanas. Los miembros de la Comisión de I niciativas Anti-C orrupción (ACSC) crean, para cada área, subcomisiones quetrabajan en el desarrollo de propuestas políticas. Las subcomisiones utilizan los resultados de los diagnósticos y las mejores experiencias dereformainternacionales para orientar sus propuestas.

El trabajo de las subcomisiones se complementa a veces con las iniciativas piloto, que pueden proporcionar resultados tangibles para una población prudente. Los programas piloto prome ten generar una confianza social y un apoyo político que son necesarios para el éxito del programa anti-corrupción. Cuando consecuentemente se revela el plan nacional, la C omisión de Iniciativas Anti-Corrupción puede demostrar que ya está trabajando en una reforma concreta, y que no está solamente shaciendo otro informe». El hecho de que la Comisión de Inicativas AntiCorrupción esté jugando un papel activo en la implantación de las reformas anti-corrupción fortalece la recepción del plan nacional.

Para ser consideradas en la selección, las instituciones piloto necesitan la presencia de un líder fuerte y ético, acompañado de la voluntad de los funcionarios públicos de aceptar a la sociedad civil con capacidad para monitorizar y evaluar. En Paraguay, la Comisión de Iniciativas Anti-Corrupción desarrolló programas piloto en tres instituciones. Un programa se ocupaba de los delitos financieros de la oficina del fiscal, y estaba dirigido por el Subcomité Judicial de la Comisión de Iniciativas Anti-Corrupción. El segundo programa controlaba las reformas asumidas en dos oficinas de aduanas (Ciudad del Este y Asunción), bajo la jurisdicción del Subcomité de Aduanas de la Comisión de Iniciativas Anti-C orrupción. El tercer programa revisó tres leyes consideradas como necesarias en la lucha contra la corrupción, bajo la dirección del Subcomité de G estión. Una vez que los programas están en su sitio, los subcomités controlan el progreso cada tres meses, revisando la información, y la percepciones del usuario acerca de la eficiencia, la efectividad y la corrupción.

A lo largo de está fase de planificación, el WBI proporciona asistencia técnica a los subcomités en su trabajo esencial, y entrena a miembros del comité para sus papeles y responsabilidades. Algunos de esos papeles y responsabilidades incluyen el uso de instrumentos de diagnóstico para priorizar y secuenciar las reformas dentro de las instituciones, la preparación para el Taller $\mathrm{Na}$ cional de Integridad, la solicitud de puntos de vista alternativos (por ejemplo, de partidos políticos, de $0 \mathrm{~N} G \mathrm{~s}$, y de los medios de comunicación) y el control de la implantación de la reforma en concordancia con el plan de acción finalizado. La asistencia varía en función de la petición específica y de las necesidades del país, y puede incluir (el) proporcionar talleres para periodistas, reuniones con los donors, facilitar el entrenamiento para grupos de trabajo acerca de cómo usar los instrumentos para el diagnóstico, y proporcionar diferentes experiencias y herramientas para luchar contra la corrupción en distintas áreas (como, por ejemplo, en la administración de justicia, las aduanas y la gestión).

D urante la fase preparatoria, la Comisión de Iniciativas se re úne también con los diferentes donors para desarrollar nexos de colaboración en las áreas de anti-corrupción. Los donors están invitados a participar en un proceso que pertenece a los países de los clientes. Estas reuniones se utilizan para aumentar el grado de compromiso del donor en el plan de acción, implicándoles en el proceso y preguntando sus puntos de vista. Las reuniones también ayudan a coordinar la distribución de los escasos recursos.

\section{Plan de acción y el taller nacional}

Una vez terminada la fase preparatoria, el país celebra un taller nacional para discutir el plan de acción preliminar, junto con los resultados de los estudios y el trabajo de la Comisión de Iniciativas. A este taller acuden todos los stakeholders más importantes, incluyendo a la sociedad civil, a las empresas, y a los repre sentantes del gobierno, y está abierto a los medios de comunicación. El taller presenta un plan de acción consensuado, al que puedan comprometerse tanto el gobierno como la sociedad civil, y que lleve a la formación de equipos de trabajo que revisen las reformas propuestas en las áreas específicas. El taller también ayuda a aumentar el conocimiento del público general de los costes económicos y sociales de la corrupción, así como de las reformas necesarias para prevenir y combatir la corrupción.

Paraguay, por ejemplo, celebró un taller nacional en diciembre de 2000, donde el Presidente G onzález M acchi se comprometió publicamenteaimplantar un programa anti-corrupción en su país. En un discurso dijo que «el primer paso para luchar contra la corrupción es reonocer que existe corrupción». Respaldó el plan de acción que la Comisión de Iniciativas había formulado duranteel año anterior, uniéndoseal Presidente de la CorteSupremay al Pre sidentedel Congreso, firmando el compromiso deadoptar un plan de acción delante de un público de 700 personas, incluyendo ministros, sociedad civil, donorsy los medios de comunicación. La prensa y televisión local dedicaron mucho espacio acerca del programa, del plan de acción y del discurso del presidente. El hecho 
de queel proceso sea transparente, de que exista compromiso político para la reforma, la capacidad técnica para ejecutar las reformas y la participación de la sociedad civil, ha dado credibilidad al plan deacción, y ha autorizado a los stakeholderspara que controlen el proceso. La participación de los donorsen el proceso ha asegurado el apoyo para el programa deacción.

\section{El control y la evaluación}

M ás allá del control interno de la Comisión de Iniciativas, el programa anti-corrupción utiliza instrumentos externos de control. El Instituto del Banco M undial facilita seminarios que piden una retroalimentación por parte de los participantes acerca de la implantación de las reformas. El gobierno obtiene información valiosa, y las habilidades para determinar cómo puede ser mejorada 0 adaptada la reforma dentro de las diferentes agencias.

Siguiendo a la fase de implantación, se dirige un segundo estudio diagnóstico para evaluar el impacto de la reforma cada dos 0 tres años. Comparando los indicadores de actuación y percepción, que miden la eficiencia, el acceso, y la transparencia, con el diagnóstico inicial, aquellos que están implicados pueden ver qué zonas muestran mejoría y por qué.

La retroalimentación preliminar de los talleres y los estudios en Bolivia y Paraguay muestra que las metodologías basadas en el consenso utilizadas para implantar reformas institucionales, sirven para aumentar la transparencia, el acceso público a los servicios y la capacidad del gobierno de enfocar los problemas. Con el comienzo de un programa participativo, la sociedad civil se está convirtiendo en una parte integral y activa en la decisión y en el proceso público de la toma de decisiones en estos países. D ebido a que los ciudadanos están implicados en definir las prioridades y en determinar las alternativas, ahora exigen eficiencia en la ejecución de los proyectos. Como stakeholders, han sido capacitados para preocuparse por los proyectos y controlar su actuación.

\section{Lecciones aprendidas hasta la fecha}

Estos acercamientos son relativamente nuevos desarrollos, $y$, en la mayoría de los países donde se han adoptado, el proceso ni ha seguido su curso, ni ha sido aplicado a conciencia. El proceso en sí mismo lleva mucho tiempo para generar resultados signifi- cativos (10 a 15 años). Sin embargo, a nivel municipal, se puede observar el impacto en un período tan breve como dos años 3 .

Las lecciones aprendidas de estos esfuerzos están sujetas a excepciones, y generalmente supone que existe una particular ventana para la oportunidad en el pás, para iniciar un cambio a largo plazo a través de un proceso participativo.

- El compromiso político es indispensable. En todos los niveles del gobierno, ésta es una condición crucial para alcanzar unas reformas institucionales comprensibles, especialmente para crear sistemas fiables y transparentes y para erradicar la corrupción. D esarrollar eimplantar exitosamente una infraestructura integrada para luchar contra la corrupción a nivel nacional no es posible sin el compromiso político local.

- Los estudios son un método útil para incrementar la conciencia del hecho y para diseñar, controlar y evaluar las re formas. Los estudios de diagnóstico son instrumentos efectivos para identificar problemas específicos que están correlacionados con la corrupción. Para dar credibilidad al esfuerzo de la reforma, el estudio debería estar dirigido por consultores independientes.

- La metodología usada en este programa utiliza innovadores acercamientos participativos para garantizar el consenso entre el gobierno y la sociedad civil. Los estudios recogen percepciones generales sobre la corrupción, y les da voz a los stakeholders en la identificación de los problemas. La metodología participativa utilizada en los talleres aumenta, entonces, la transparencia y la credibilidad del proceso de toma de decisiones. Los ciudadanos entienden que poniéndole punto final a los sobornos, libera los recursos financie ros que de otra forma podrían estar distribuidos en servicios sociales esenciales. Los ciudadanos pueden estar más satisfechos con los servicios y quejarse menos como resultado de entender que la prioridades y los trade offs son necesarios cuando se trabaja con un presupuesto limitado.

- El acercamiento de la construcción de coaliciones potencia la sustentabilidad de las reformas. D ebido a que una sección más amplia de la población se involucra en la definición de prioridades y en la determinación de los trade offs, exigen eficiencia en la ejecución de los proyectos. Como stakeholders, están capacitados para preocuparse por los proyectos y controlar su actuación. A su vez, ha aumentado la propensión a la colaboración ciudadana en el gobierno.

- La experiencia demuestra los poderosos y beneficiosos efectos de una innovadora mezcla de voluntad política local, de capacidad técnica para ejecutar reformas, y de un fuerte compañerismo con la sociedad civil en el empuje para potenciar la eficiencia, la equidad y la transparencia en un país. 
Artículo traducido por Laura AlBa M uñoz.

* Instituto del Banco M undial.

${ }^{1}$ En Latinoamérica, los jefes de Estado de Paraguay, Bolivia, Ecuador, Nicaragua, Colombia y H onduras han solicitado dicha ayuda de la donor community.

${ }^{2}$ Después de que Raúl Cubas Grau fuera elegido presidente en mayo de 1998, su vicepresidente, Luis M arís Argaña, fue asesinado en marzo de 1999. Debido a sospechas acerca de su implicación, el Presidente Cubas fue obligado a dejar el cargo el 28 de marzo, y le sucedió Luis Ángel González Macchi, el presidente del Senado. Las débiles instituciones democráticas paraguayas sobrevivieron a una crisis política y una abrupta transferencia de poder.

${ }^{3} \mathrm{~A}$ nivel municipal, ha habido varias experiencias positivas. El programa de Campo Elías, por ejemplo, fue diseñado para ayudar a ani mar a un gobierno municipal eficiente, crébley transparente en Venezuela eimplica un trabajo conjunto con el W BI, el alcaldedela ciudad y la sociedad civil. El programa comenzó en abril de 1998 y duró hasta noviembrede 1999. Uno delos objetivos del programa era la distribución delos recursos municipales de una forma más eficiente y ecuánime, luchando contra la corrupción. Anteriormente, los recursos se agotaban debido a prácticas corruptas. Para dar prioridad a lasáreas parala reforma, el alcal dey la sociedad civil utilizaron los grupos de trabajo participativos, y desarrollaron un plan deacción. Losgrupos de traba jo propusieron formasinnovadoras de afrontar los problemas defalta de información, de fiabilidad, y una exageradamente compleja infraestructura regulatoria. Los grupos identificaron los problemas, a los actores para implantar las reformas, los resultados esperados, y las soluciones propuestas. El programa utiliza distintas medidas para potenciar la fiabilidad, la transparenciay la credibilidad. El éxito del programa supuso una redistribución de los recursos y una menor corrupción municipal, la cual fue medida usando un estudio de una unidad familiar al final del programa. 